\title{
Produção brasileira da Ciência da Informação na Web of Science entre 1994 e 2013 e a lista Qualis/Capes da Área
}

La producción brasileña sobre Ciencias de la Información en la Web of Science (1994-2013)

y la lista de áreas Qualis/CAPES

Brazilian Information Science production in Web of Science (1994-2013) and the Qualis/CAPES area list

\section{Adilson Luiz Pinto (1), Márcio MAtias (2), José Antonio MoReiro GonZÁlez (3)}

$(1,2)$ Universidade Federal de Santa Catarina, Brasil; didis98@hotmail.com, matias97@gmail.com

(3) Departamento de Biblioteconomía y Documentación, Universidad Carlos III de Madrid, España, jamore@bib.uc3m.es

\begin{abstract}
Resumen
Se visualiza la producción científica brasileña en las revistas de ciencia de la información incluidas en la Web of Science. Se procesan 58 revistas y se muestra el contraste con la lista Qualis/CAPES. Se buscaron los artículos sobre Ciencias de la Información y Bibliotecología con al menos un autor brasileño entre 1994 y 2013. Los resultados muestran las revistas internacionales relevantes para los investigadores brasileños, y los autores más productivos. La red de producción científica concentra sólo trece títulos en la lista de áreas Qualis/Área. De las veinte revistas más citadas, quince no son parte de dicha lista. Como conclusión, la lista de áreas Qualis no promueve que los investigadores brasileños publiquen en las mejores revistas del área de la Web of Science.
\end{abstract}

Palabras clave: Bibliometría. Políticas científicas. Producción científica. Web of Science. Mapas de ocurrencia. Mapas de autoría. Mapas de revistas.. Mapeo por referencia. Qualis/CAPES.

\section{Introdução}

A avaliação da qualidade da produção bibliográfica tem sido um dos principais aspectos que compõe o processo de avaliação de programas de pós-graduação.

No Brasil, a Coordenação de Aperfeiçoamento de Pessoal de Nível Superior (Capes) é o órgão do Ministério da Educação do Brasil responsável pelo reconhecimento e a avaliação de cursos de pós-graduação stricto-sensu (mestrado profissional, mestrado acadêmico e doutorado) em âmbito nacional. O CAPES assumiu o compromisso de desenvolver procedimentos para diferenciar a qualidade da produção bibliográfica dos programas de pós-graduação stricto sensu (mestrado e doutorado), denominada de Qualis. Este sistema foi gerado por um estrato de qualidade dessa produção a partir da classificação dos periódicos utilizados na sua divulgação

\begin{abstract}
A visualization of Brazilian scientific production in journals of Information Science included in Web of Science is provided. It involves 58 journals and shows the contrast with Qualis/CAPES list. Articles on Information Science and Library Science with at least one Brazilian author, from 1994 to 2013, were searched. The results show that these international journals are relevant for brazilian researchers, and the most productive authors. The scientific production network concentrates only 13 titles in Qualis/Capes Area list. From the most cited 20 journals, 15 are not part of this list. As a conclusion, the Qualis Area list don't encourage the Brazilian researchers to publish in the most of the best journals of the area in Web of Science.
\end{abstract}

Keywords: Bibliometrics. Scientific policies. Scientific production. Web of Science. Occurrence maps. Author mapping. Journals mapping. Reference mapping. Qualis/CAPES.

pelos pesquisadores de cada área do conhecimento (CAPES, 2007; 2008; 2013).

Os critérios para a classificação no Qualis são diferenciados entre as áreas do conhecimento e são determinados por comissões de consultores destas áreas. A partir da aplicação destes critérios, as publicações são contempladas em uma listagem estratificada, e passa a ser incorporada em uma base para o processo de avaliação da produção dos programas nacionais de pósgraduação.

Como envolve fator determinante no processo de avaliação destes cursos, as características e as mudanças nos procedimentos do Qualis têm sido discutidas, e muitas vezes questionadas pelos responsáveis que editam as publicações (editores e gestores) e pelos pesquisadores que submetem os estudos nas mais diversas áreas do conhecimento, onde discutem a indução 
deste sistema, como o caso para a Psicologia (Jacon, 2007); a necessidade de uma renovação mais igualitária para as áreas (Dupont; Dias, 2008); o não cumprimento de uma geração de indicadores padronizados (Rocha-ESilva, 2009; Silva, 2009); a necessidade de olhar com outros olhos a área de Engenharias (Lins; Pessoa, 2010) e um papel social a este tipo de avaliação (Leite; Codato, 2013).

$\mathrm{Na}$ área de Ciência da Informação, o cenário internacional de periódicos científicos apresenta um número considerável de títulos influentes com características direcionadas ao contexto social, humano e tecnológico desta área do conhecimento.

Diante deste importante sistema, este estudo objetiva: (1) identificar os periódicos de contexto internacional em que os autores brasileiros publicam, segundo uma busca avançada pelo campo área do conhecimento na Web of Science; (2) averiguar os autores mais representativos; (3) identificar o índice de citação das revistas (onde os autores brasileiros publicaram), fator este que a Capes alegou como um ponto de relevância em suas avaliações, entretanto este processo não está inserido em suas listagens das áreas de Ciências Sociais Aplicadas I.

Como resultado desse trabalho, baseando-se em uma análise bibliométrica, esperou-se identificar e sugerir, listas de títulos para aumentar o índice de revistas A1 no sistema Qualis/Capes. Tal incremento poderia trazer um número mais justo de títulos com visibilidade para a área de estudo.

Ao analisar os autores mais representativos e o índice de citação, a segunda contribuição deste estudo é identificar o nível de reconhecimento e a visibilidade dos autores por seus pares, já que os artigos são publicados nos periódicos mais representativos da área da Ciência da Informação e sabemos que o índice de citação é algo ainda pendente no sistema Qualis/Capes.

\section{O Qualis}

A listagem da Capes (2013) para periódicos divididas em 49 áreas - , da forma como está desenvolvida, é conferida na visão das grandes áreas do conhecimento; até 2010/2011 sua confecção não levava o fator de indexação da revista para algumas áreas, como as Ciências Sociais Aplicadas I. Em 2012 este cenário passa por uma mudança e começam a classificar como A1 todas as revistas indexadas na Web of Science, o estrato de maior qualidade para a grande área. As revistas indexadas na Scopus ou SciELO passaram a classificação de A2. Se por acaso uma revista esteja indexada na Scopus ou SciELO ao mesmo tempo também ganha a classificação A1. As demais classificações estão concentradas em indexação de repositórios, bases de dados de referência bem como critérios de suas políticas de publicação, ranqueadas como B1, B2, B3, B4, B5 e C, dependendo do seu grau de exigência e critérios adotados.

Na primeira versão em 2005, o marco inicial do sistema Qualis/Capes, existiam critérios para a qualificação das revistas científicas, onde deveriam ter o registrado de ISSN; regras de publicação (detalhadas); procedimentos para a submissão de textos originais; Corpo editorial, de preferência com pesquisadores estrangeiros; Regularidade na periodicidade; Um controle sistemático dos últimos 5 anos; Contar com colaboradores nacionais e internacionais para avaliações (CAPES, 2005).

Entretanto, estes critérios de 2005 são pontos iniciais para a existência de uma revista científi$\mathrm{ca}$, independente da representação local, nacional ou internacional. Além disto, na área de Ciência da Informação, a categorização dos periódicos apresentou em sua história alguns aspectos que chamaram atenção: nas listas de 2005 e 2007/2009 constavam periódicos classificados como internacionais e que não estavam indexados em nenhuma base ou diretório. $\mathrm{O}$ critério de internacionalização até então era contar com avaliadores e o Conselho Editorial estrangeiro.

A versão atual do sistema para Ciência Sociais Aplicada I (2012) requer, além dos itens mencionados, o fator de impacto de periódicos, conforme aplicado a algum tempo às áreas de Química, Física e Biologia que categorizam suas publicações em periódicos ISI/JCR e SciELO (Andrade; Galembeck, 2009) em avaliações anteriores. Estes critérios somente fazem parte da avaliação a partir do momento que um autor publica neles e que o mesmo esteja atuando em um Programa de PósGraduação Stricto Sensu. Para estas áreas o critério até é válido, porém para áreas como as Humanas e Sociais o critério fica subjetivo, em especial porque o fator de impacto nestas áreas nem sempre é justo e muito menos alto. Outro detalhe é qual fator de impacto utilizar? De qual base de dados?

Pelos procedimentos atuais utilizados pela Capes, existem duas formas para um periódico ser inserido na lista Qualis de uma área.

$\mathrm{Na}$ forma mais comum, o processo é disparado pela declaração de um dos programas de pósgraduação reconhecidos pela Capes de que

Pinto, Adilson Luiz; Matias, Márcio; Moreiro González, José Antonio. Produção brasileira da Ciência da Informação na Web of Science entre 1994 e 2013 e a lista Qualis/Capes da Área. // Ibersid. 10:1 (2016) 51-61. ISSN 1888-0967. 
docentes, discentes ou pesquisadores publicaram artigos científicos no veículo científico; na Capes, o periódico passa então por um processo de padronização (verificação de dados), de classificação quanto aos atributos de qualidade, para, então, poder ser indicado pela área de avaliação para participar da lista de veículos publicados no Qualis das Áreas. A segunda forma envolve, como passo inicial, a indicação direta do título pelo Representante de Área, seguindo, a partir de então, os mesmos passos da forma anterior (CAPES, 2007; 2008; 2013).

Segundo Capes (2007), no caso da indicação direta do Representante de Área para inserção de periódico na lista Qualis de uma área, a justificativa é a sua relevância qualitativa como importante veículo de divulgação da área. Nessa situação, o Qualis está sendo utilizado como indutor de publicação de qualidade em veículos de qualidade reconhecida, independentemente desse veículo já ter sido utilizado para divulgar a produção bibliográfica de algum programa nacional.

A questão é que, na área de Ciência da Informação, diversos títulos considerados por pesquisadores como de relevância qualitativa não constam na lista Qualis desta Área. Um exemplo claro é o Journal of Documentation, que não faz parte da listagem Qualis porque não foi publicado nenhum estudo nele por pesquisadores credenciados nos programas de pós-graduação brasileiros e muito menos indicado por representantes da área para compor a lista.

Segundo Erdmann (2009) a classificação dos periódicos no Qualis/Capes tem papel indutor de onde o pesquisador deve publicar. Além disto, a avaliação da qualidade da pesquisa científica contribui para promover o desenvolvimento, orientar investimentos e induzir foco de pesquisa e veículo de publicação (Lins; Pessoa, 2010).

Mesmo que a produção em determinados veículos que não constem no Qualis da Área possa ser contabilizada no processo de avaliação de programas de pós-graduação, na verdade, os pesquisadores e os programas de pósgraduação têm focalizado, na maioria das vezes, nos veículos constantes nas listas Qualis das Áreas (CAPES, 2007). Pelo fato de determinados veículos não constarem na lista Qualis, pode-se afirmar que o sistema, desta forma, subutiliza o potencial de induzir a publicação em veículos de qualidade, na medida em que não divulga a possibilidade de publicar em veículos reconhecidos e estimula, assim, a publicação com maior frequência nos mesmos veículos.
Observem-se alguns casos como a Education for information (ISSN 0167-8329), International Journal \& Library Review (ISSN 1057-2317) e Journal of Documentation (ISSN 0022-0418), títulos influentes para a Ciência da Informação, inclusive utilizado por pesquisadores da área em suas publicações, entretanto estes títulos não estão incluídos na lista de Ciências Sociais Aplicadas I, à qual a Ciência da Informação está relacionada, porém indexados em outras áreas do conhecimento nas edições até 2009.

Nesse ambiente, cabe lembrar o que se compreende por comunicação científica. Ela pode ser definida como a troca de informação entre membros de uma comunidade científica, incluindo, de acordo com Garvey (1979), atividades relacionadas à produção, disseminação e uso da informação, do momento em que o cientista concebe a ideia para pesquisa até o momento em que os resultados desta são aceitos como constitutivos do conhecimento científico. Por meio da comunicação científica, os pesquisadores que participam nas comunidades são informados sobre as tendências da área, os estudos desenvolvidos e seus resultados.

Os periódicos científicos têm um papel importante na comunicação entre os pesquisadores. O número de publicações do grupo pode ser considerado como um indicador da sua produção científica; o número de vezes que os artigos dos grupos são referenciados nas publicações é a medida da visibilidade desses artigos. No sistema global de periódicos, é possível distinguir entre os periódicos centrais internacionais e os periféricos, categorizados como regionais. A Thomson Reuters, por meio de suas bases de dados indexadas na Web of Science, engloba a maioria dos periódicos internacionais.

Uma das atividades da Web of Science é a análise da produção científica, que atualmente é cada vez mais frequente nos estudos de comunicação científica, sendo parte desse grande sistema social, em que

[...] a ciência necessita ser considerada como um amplo sistema social, no qual uma de suas funções é disseminar conhecimentos. Sua segunda função é assegurar a preservação de padrões, e a terceira é atribuir créditos e reconhecimento para aqueles cujos trabalhos têm contribuído para o desenvolvimento das idéias em diferentes campos. (Macias-Chapula, 1998, p. 136)

Estes dois ambientes, a listagem da Qualis/Capes e a indexação nas bases internacionais, como a Web of Science, fazem com que sua mensuração seja exigida. Esta medição passa diretamente pela Bibliometria, que se 
baseia em julgamentos quantitativos, com aplicação e métodos adotados da estatística e matemática para analisar a informação e a comunicação, bibliográfica ou documental. Sua aplicação pode ser para avaliar a atividade, a produção ou progresso. E seu propósito está voltado em trabalhar com entradas (input) de dados/informação e sua geração em forma de saídas ou produtos (output).

Por sua vez, os indicadores de input e output, assim como suas relações e importância para os estudos quantitativos da ciência, são tratados por Spinak (1998) como insumo e resultados para a atividade científica, seja de instituição ou de uma empresa.

De acordo com Poblacíon e Oliveira (2006, p. 68), "input é uma combinação dos fatores que viabilizam a produção de determinada quantidade de bens e serviços (output)". A mensuração torna-se a base para produção de indicadores científicos.

\section{Metodologia}

Este é um estudo quantitativo/descritivo aplicado à linha temporal que transcorre de 1994 a
2013. Selecionaram-se 742 registros/artigos referentes às áreas de Biblioteconomia e Ciência da Informação de pesquisadores de instituições brasileiras, segundo a base de dados Web of Science, baseando-se na cientografia e análises de redes produtivas proposta por Leydesdorff (2007), que retrata a produção e citação por grafos.

Este estudo aborda redes de produção e citação de periódicos científicos, segundo seu fator de influência na rede, onde a mesma foi desenvolvida em dois passos: um para explicar a estratégia de busca, e outro para recuperação da informação e tratamento dos dados pertinentes, mensurados por estatísticas e teorias bibliométricas.

O padrão estratégico para busca de dados e recuperação caracterizou-se por uma pesquisa avançada pelo campo Source Title [SU=information science and library science]. Depois foi determinada a produção brasileira pelo refinamento, que está dividida entre 1.334 autoridades. Dentro do refinamento, também identificamos os periódicos nos quais os brasileiros publicaram seus trabalhos:

\begin{tabular}{|c|c|c|}
\hline Revistas & País da revista & Editora \\
\hline Aslib Proceedings & Reino Unido & Emerald \\
\hline Electronic Library & Reino Unido & Emerald \\
\hline Government Information Quarterly & Reino Unido & Elsevier \\
\hline Health Information and Libraries Journal & Estados Unidos & Wiley \\
\hline IFLA Journal-International Federation of Library Associations & Holanda & IFLA \\
\hline Informação \& Sociedade-Estudos & Brasil & UFPB \\
\hline Informacios Tarsadalom & Hungria & Infonia \\
\hline Information \& Culture & Estados Unidos & JSTOR \\
\hline Information \& Management & Holanda & Elsevier \\
\hline Information and Organization & Reino Unido & Pergamon \\
\hline Information Development & Reino Unido & Sage \\
\hline Information Processing \& Management & Reino Unido & Elsevier \\
\hline Information Research-An International Electronic Journal & Reino Unido & Univ. Sheffield \\
\hline Information Society & Estados Unidos & Taylor \& Francis \\
\hline Information Systems Research & Estados Unidos & INFORMS \\
\hline Information Technology \& Management & Holanda & Elsevier \\
\hline Information Technology \& People & Reino Unido & Emerald \\
\hline Information Technology and Libraries & Estados Unidos & ALA \\
\hline Information Technology for Development & Reino Unido & Routledge \\
\hline International Information \& Library Review & Reino Unido & Taylor \& Francis \\
\hline International Journal of Computer-Supported Collaborative Learning & Estados Unidos & Springer \\
\hline International Journal of Geographical Information Science & Reino Unido & Taylor \& Francis \\
\hline International Journal of Information Management & Reino Unido & Pergamon \\
\hline Investigacion Bibliotecologica & México & UNAM \\
\hline Journal of Documentation & Reino Unido & Emerald \\
\hline Journal of Education for Library and Information Science & Estados Unidos & ALISE \\
\hline Journal of Global Information Management & Estados Unidos & IGI Global \\
\hline Journal of Health Communication & Estados Unidos & Taylor \& Francis \\
\hline Journal of Information Science & Reino Unido & Sage \\
\hline
\end{tabular}




\begin{tabular}{|c|c|c|}
\hline Journal of Information Technology & Reino Unido & Palgrave Macmillan \\
\hline Journal of Informetrics & Holanda & Elsevier \\
\hline Journal of Knowledge Management & Reino Unido & Emerald \\
\hline Journal of Librarianship and Information Science & Reino Unido & Sage \\
\hline Journal of Scholarly Publishing & Canada & Univ. Toronto \\
\hline Journal of Strategic Information Systems & Holanda & Elsevier \\
\hline Journal of the American Medical Informatics Association & Reino Unido & Univ. Oxford \\
\hline Journal of the American Society for Information Science and Technology & Estados Unidos & ASIS\&T \\
\hline Journal of the Medical Library Association & Estados Unidos & Medical Library Ass. \\
\hline Knowledge Management Research \& Practice & Reino Unido & Palgrave Macmillan \\
\hline Knowledge Organization & Alemanha & Ergon Verlag \\
\hline Library Collections Acquisitions \& Technical Services & Reino Unido & Taylor \& Francis \\
\hline Libri & Alemanha & De Gruyter Saur \\
\hline Mis Quarterly Executive & Estados Unidos & Indiana Univ. \\
\hline Perspectivas em Ciencia da Informacao & Brasil & UFMG \\
\hline El Profesional de la Informacion & Espanha & EPI \\
\hline Program-Electronic Library and Information Systems & Reino Unido & Emerald \\
\hline Publishing Research Quarterly & Estados Unidos & Springer \\
\hline Research and Advanced Technology for Digital Libraries & Alemanha & Springer/Verlag \\
\hline Research Evaluation & Reino Unido & Univ. Oxford \\
\hline Restaurator & Alemanha & De Gruyter Saur \\
\hline Revista Espanola de Documentacion Cientifica & Espanha & CSIC \\
\hline Scientist & Estados Unidos & LabX Media Group \\
\hline Scientometrics & Holanda & Elsevier \\
\hline Serials Review & Reino Unido & Taylor \& Francis \\
\hline Social Science Computer Review & Estados Unidos & Sage \\
\hline Social Science Information & Reino Unido & Sage \\
\hline Telecommunications Policy & Reino Unido & Pergamon \\
\hline Transinformacao & Brasil & PUC-Campinas \\
\hline
\end{tabular}

Tabela I. Revista onde publicaram os autores brasileiros na Web of Science (1994/2013) (Fonte: Dados da pesquisa, 2014. Extraído da Web of Science e do UlrichsWeb)

Para o tratamento da informação, utilizou-se a estratégia de mensurar a produção e a citação por meio de mapas de relacionamento (grafos), focadas na análise de redes sociais, visando o uso da medida radial. A medida radial toma como ponto de referência um nó dado, contabilizando seu percurso pela rede desde o inicio até o fim da sua trajetória Esse tipo de análise, também conhecida como visualização da informação favorece a uma representação dos dados mais interativos.

No que concerne o tratamento dos registros recuperados, utilizou-se Infotrans para a análise de conteúdo, um programa para reformatação de dados desenvolvido por luK Rieth $\mathrm{GmbH}$ Freiburg. Para a visualização da informação, utilizou-se o CiteSpace (Chen, 2006), que trata e define os índices de ocorrências automaticamente.

A matrix para trabalhar estes dados foi desenhada simetricamente, pensando co-ocorrências (de autoria e de citação) e, sua aplicação foi para identificar a centralidade de grau, recurso que os software pode gera automático.

\section{Resultados e discussões}

Esse tópico objetivou investigar o output nos mais importantes periódicos de áreas correlatas à Ciência da Informação e a visibilidade destes na Web of Science.

Dentre esses periódicos, respeitando o período de estudo que transcorreu entre 1994-2013, identificou-se que somente $13(22,41 \%)$ de 58 revistas estavam incluídos na listagem da Qualis/Capes (Electronic Library, Informação \& Sociedade: Estudos, Information Development, International Information \& Library Review, Investigación Bibliotecológica, Journal of Strategic Information Systems, Journal of the American Society for Information Science and Technology, Journal of the Medical Library Association, Knowledge Organization, Perspectivas em Ciência da Informação, Profesional de la Información, Scientometrics e Transinformação. 


\begin{tabular}{|c|c|c|c|}
\hline Revistas & $F R$ & $\%$ & Bradford \\
\hline Informação \& Sociedade-Estudos & 188 & 25,34 & $1^{\circ}$ Núcleo \\
\hline Perspectivas em Ciencia da Informacao & 179 & 24,12 & $1^{\circ}$ Núcleo \\
\hline Transinformacao & 105 & 14,15 & $2^{\circ}$ Núcleo \\
\hline Scientometrics & 63 & 8,49 & $2^{\circ}$ Núcleo \\
\hline Journal of the American Society for Information Science and Technology & 17 & 2,29 & $2^{\circ}$ Núcleo \\
\hline Information Processing \& Management & 16 & 2,16 & $2^{\circ}$ Núcleo \\
\hline Social Science Information Sur Les Sciences Sociales & 16 & 2,16 & $2^{\circ}$ Núcleo \\
\hline Knowledge Organization & 15 & 2,02 & $2^{\circ}$ Núcleo \\
\hline Journal of the American Medical Informatics Association & 9 & 1,21 & $2^{\circ}$ Núcleo \\
\hline Investigacion Bibliotecologica & 8 & 1,08 & $2^{\circ}$ Núcleo \\
\hline International Journal of Geographical Information Science & 8 & 1,08 & $2^{\circ}$ Núcleo \\
\hline 47 revistas representando $15,90 \%$ & 118 & 0,94 & $3^{\circ}$ Núcleo \\
\hline TOTAL (58 REVISTAS) & 742 & 100,00 & \\
\hline
\end{tabular}

Tabela II. Núcleo das revistas segundo a lei de dispersão de Bradford (Fonte: Dados da pesquisa, 2014. Extraído da Web of Science.

Para a aplicação da lei de dispersão de Bradford, utilizamos a regra de aproximação nos valores básicos da lei $\left(1: n: n^{2}\right)$, onde o primeiro núcleo contou com duas revistas (Informação \& Sociedade-Estudos com $\mathrm{n}=188$ artigos e Perspectivas em Ciencia da Informacao com $\mathrm{n}=179$ artigos), tendo um percentual de $49,46 \%$ de todo output estudado. No segundo núcleo tivemos 9 revistas (Transinformacao com $\mathrm{n}=105$ artigos; Scientometrics com $\mathrm{n}=63$ artigos; Journal of the American Society for Information Science and Technology com $\mathrm{n}=17$ artigos; Information Processing \& Management e Social Science Information Sur Les Sciences Sociales ambas com $n=16$ artigos; Knowledge Organization com $\mathrm{n}=15$ artigos; Journal of the American Medical Informatics Association com $\mathrm{n}=9$ artigos; Investigación Bibliotecológica e International Journal of Geographical Information Science ambas com $n=8$ artigos) totalizando $34,64 \%$ das produções. E no 3 núcleo um total de 47 revistas, representando $15,90 \%$ dos outputs.

O notório nesta análise é que somando as revistas brasileiras indexadas na Web of Science nesta análise o percentual de produção fica em $79,64 \%$, representas pelas duas revistas do $1^{\circ}$ núcleo e a primeira revista do $2^{\circ}$ núcleo. $\mathrm{O}$ que isso representa é que mesmo os brasileiros tendo representatividade dentro da Web of Science suas produções ainda estão no idioma português, não justificando um processo total de internacionalização de seus outputs.

Dentro do contexto de análise para a listagem Qualis/Capes, foram identificadas as principais áreas de conhecimento que este sistema gera para as revistas estudadas, sendo vislumbrada na figura 1 , na página seguinte.
O grande destaque deste universo são as revistas Web of Science sem áreas de conhecimento para a listagem Qualis/Capes, porém que detém publicação considerável, localizada na caixa abaixo a direita (representada pelos círculos vermelhos). $O$ isso quer representar é que se publica consideravelmente nestes periódicos, porém as Ciências Sociais Aplicadas I (área geral em que a Ciência da informação faz parte na listagem) não as pontuam porque nestas publicações não tiveram um autor que faça parte de algum Programa de Pós-Graduação em Ciência da Informação do Brasil.

Outro detalhe fica pela parte central da figura 1, onde aparecem as áreas (i) Interdisciplinar; (ii) Administração, Ciências Contábeis e Turismo; e (iii) Ciência da Computação, como Journal of Documentation, ASLIB Proceeding, Research Evaluation, Information Processing \& Management, Information Technology and Libraries entre outras.

Então, o que realmente é pontuado para a área da Ciência da Informação pela listagem Qualis/Capes em Ciências Sociais Aplicadas I são as revistas em azul. Isso ocorre pelo simples fato de algum autor brasileiro fazer parte de algum programa de pós-graduação na área de Ciência da Informação.

Desta forma, entendemos que o grande problema do sistema Qualis/Capes está em reproduzir a publicação dos autores brasileiros como sendo a mais relevante para as suas respectivas áreas, ocasionando o fato de uma revista ser muito importante para a área somente pelo fato de ter artigos publicados pelos pesquisadores brasileiros, no qual não deveria ser um padrão e sim um indicativo. 




Figura 1. Distribuição das revistas segundo suas áreas de conhecimento pela categorização Qualis/CapesFonte: Dados da pesquisa, 2014. Extraído da Web of Science, trabalhado em CreatePajek e NetDraw.

Acreditamos que a listagem necessita de um ajuste no sentido de não deixar de contemplar revistas chave para a área internacionalmente, como é o caso da Journal of Documentation, Aslib Processing e Information Processing \& Managent, mesmo que um autor brasileiro não publique nelas.

Por outro lado, é necessário computar a produção brasileira em autoridades. Frente ao universo recuperado de conteúdo sobre a área de conhecimento em Ciência da Informação, foi possível identificar 1.334 autores diferentes, dos quais estiveram reunidos em 742 artigos científicos, com uma média de co-autoria de 1,79\%. Para vislumbramos esta representação utilizouse a Lei de Lotka, tendo $20 \%$ dos autores publicado $42,91 \%$ de output. Esse índice revela uma produção nacional muito igualada, com poucos destaques dentro na análise, como: M. A . Gonçalves e J. Leta (ambas com $n=15$ trabalhos); A. H. F. Laender ( $n=13)$; LS Bufrem $(n=10)$; BV Cendón ( $n=9)$; M. S. L. Fujita e R. S. Rodrigues ( $n=8$ ambas); R. R. Barbosa, A. D. da Silva, E. S. de Moura, I. M. Freire e B. RibeiroNeto (todos com $n=7$ artigos); M. B. Almeida, M. D. Aquino, C. A. A. Araújo, M. V. da Cunha, E. A. Fox, J. A. C. Guimarães, L. A. Joia, M. Oliveira, R. R. Souza e J. Wainer (todos com $n=6$ ); A. E. Arboit, L. Café, L. D. Costa, M. B. da Cunha, E. L. da Silva, F. J. N. da Silveira, F. D. de Sou- za, D. Flores, G. A. B. D. Lima, C. H. Marcondez, M. S. Nagano, J. C. R. Pereira, F. A. Ramalho, N. Reinhard, N. A. Ribeiro, M. I. Tomáel e N. Ziviani (todos com $n=5$ ). Como complemento a esta análise, vislumbramos também a elite dos autores (Price, 1963), representado por 60 autores, na figura 2 ficam expostos os com maior destaque.

$\mathrm{Na}$ análise de produção é possível perceber a centralidade de grau de alguns autores e de seus colaboradores, ressaltando-se que quanto mais visibilidade profissional o autor tem maior é a oportunidade que o mesmo tenha seus trabalhos aceitos para publicação, onde "altos índices de publicação estão fortemente relacionados ao reconhecimento e distinções científicas" (Valchy, 1970). A centralidade de grau é a medida de centralidade mais simples, onde corresponde ao número de enlaces que possui um nó com os demais. Na figura apresentada temos períodos históricos de 20 anos, onde o amarelo mais claro representa a década de 1990, a amarela mais escura a década de 2000 e o laranja, a década de 2010.

Outra análise realizada foi a análise de citação, identificando os periódicos mais referenciados, visto que este tipo de análise é algo proposto pela listagem Qualis/Capes e que não se consegue colocar em prática, somente com algumas áreas do conhecimento que determinam a 


\section{Maria Ines Tomael} Solange Puntel Mostafa

da Silveira Fabricio Jose Nascimento

\section{Jacques Wainer \\ Mauricio Barcellos Almeida \\ Beatriz Valadares Cendon \\ Barbara Coelho Neves \\ Daniel Flores

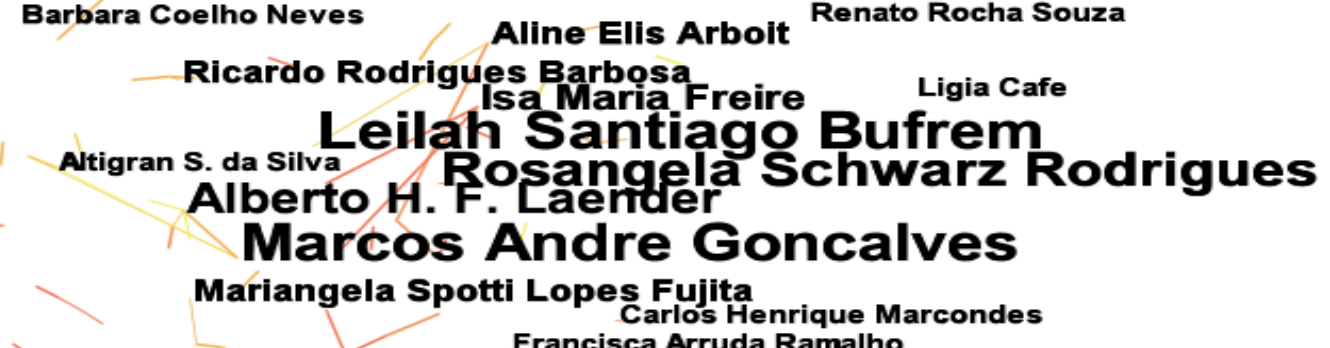 \\ Francisca Arruda Ramalho}

Souza Francisco das Chagas de

Luiz/Antonio Joia

Figura 2. Centralidade de grau dos autores mais representativos

(Fonte: Dados da pesquisa, 2014. Extraído da Web of Science e Tratado em CiteSpace)

Vislumbrando este tipo de análise determinamos o índice de dispersão das revistas citadas na nossa análise, onde o número de periódicos citados foi de 9.864 , de um total de 18.054 citações no geral (livros, teses e eventos científicos), comprovando que boa parte das referências dizem respeito a veículos científicos de difusão mais rápida. Para esta análise foram computadas todas as citações, não excluindo as autocitações.

Utilizamos para esta análise a lei de Bradford, que se aplica em 3 núcleos, onde: o primeiro reuniu $33,48 \%$ do universo (258 revistas com mais de 8 citações); o segundo núcleo com $30,35 \%$ (3768 revistas com frequência até 2 citações), e; o último núcleo com 36,17\% (6530 revistas, todas com somente 1 citação).

Em paralelo foi complementada esta análise com a elite dos periódicos, utilizando as citações da revista com maior frequência (Ciência da Informação $=518$ citações) elevada à raiz quadrada, totalizando um universo com igual ou maior de 22,59 citações, arredondado na nossa análise entre 22 citações.

\begin{tabular}{lcc}
\hline \multicolumn{1}{c}{ Revistas Citadas } & ${ }^{*} F R$ & Qualis CSA I \\
\hline Ciencia Da Informacao & 518 & B1 \\
\hline Scientometrics & 446 & A1
\end{tabular}

\begin{tabular}{lll}
\hline Perspect Cienc Inf & 201 & A1 \\
\hline J Am Soc Inform Sci & 157 & A1 \\
\hline Datagramazero & 131 & B1 \\
\hline J Am Soc Inf Sci Tec & 111 & A1 \\
\hline J Doc & 103 & \\
\hline Transinformacao & 103 & A1 \\
\hline Res Policy & 95 & \\
\hline Inform Process Manag & 91 & \\
\hline Annu Rev Inform Sci & 72 & \\
\hline Science & 69 & \\
\hline Strat Manag J & 68 & \\
\hline Commun Acm & 67 & \\
\hline Mis Quart & 64 & \\
\hline Nature & 64 & \\
\hline J Inform Sci & 62 & \\
\hline Manage Sci & 53 & \\
\hline Encontros Bibli & 48 & B1 \\
\hline Acad Manage Rev & 47 & \\
\hline Inform Soc-Estud & 47 & A1 \\
\hline Organ Sci & 44 & \\
\hline Rev Bibliotec & 44 & \\
\hline Admin Sci Quart & 43 & \\
\hline Harvard Bus Rev & 38 & \\
\hline Rev Bras Bibli & 35 & \\
\hline Knowl Organ & 34 & \\
\hline J Knowl Manag & 33 & \\
\hline Libr Trends & 32 & \\
\hline Calif Manage Rev & 31 & \\
\hline Inform Res & 31 & \\
\hline Inform Soc & & \\
\hline
\end{tabular}




\begin{tabular}{lll}
\hline Knowl Manag & 31 & \\
\hline P Natl Acad Sci Usa & 31 & \\
\hline J Informetr & 30 & \\
\hline Rev Digital Bibliote & 29 & B1 \\
\hline Acad Manage J & 28 & \\
\hline J Manage Stud & 27 & \\
\hline J Marketing & 26 & \\
\hline J Med Libr Assoc & 26 & \\
\hline Lect Notes Comput Sc & 26 & B1 \\
\hline Soc Rede & 26 & \\
\hline Org Conhec Com & 25 & \\
\hline Brit Med J & 23 & \\
\hline J Manage Inform Syst & 23 & \\
\hline J Marketing Res & 23 & \\
\hline Rev Acb Bibliotec & 23 & B2 \\
\hline Ind Corp Change & 22 & \\
\hline J Am Med Inform Assn & 22 & \\
\hline J Oper Manag & 22 \\
\hline Res Evaluat & 22 \\
\hline
\end{tabular}

Tabela III. Índice de citações da elite de periódicos (Formula 518 ل) (Fonte: Dados da Pesquisa, 2014) Nota: *FR = Frequência; Qualis CSA I = Qualis de Ciências Sociais Aplicadas I referente a 2012

Mesmo considerando somente os 51 periódicos mais relevantes, é também importante mencionar alguns títulos que, apesar de não estarem dispostos no quadro anterior, pertencem à primeira zona de dispersão de Bradford, sejam eles: Decision Sci.; Inform. Technol. Libr.; Online, Information Management; Reference Services R; Lect Notes Comput Sc; Reference Librarian; Information Seeking; J. Strategic Inf. Syst. entre outros.

Observou-se que alguns dos periódicos investigados neste estudo, segundo a análise, apresentam alto índice de citações, o que enfatiza o fato de que periódicos indexados na mesma base de dados tendem a ser mutuamente citados, gerando bom relacionamento. Entretanto, como se trata de um universo brasileiro de pesquisa também é possível identificar revistas que não estão indexados em bases de dados, porém que detêm influência na comunidade científica da área de Ciência da Informação brasileira, como a revista Ciência da Informação do IBICT, DataGramaZero, Encontros Bibli e Revista de Biblioteconomia.

Considerando a categorização central das citações, pode-se dizer que 14 periódicos têm o status de mais influentes, destacados em rosa na Figura 3: JASIST, Communication ACM, Information Processing \& Management, Research Policy, Perspectivas em Ciência da Informação, Journal of Management Studies, ARIST, Journal Management Information System; Lecture Notes Computer Science, Ciência da Informação, MIS Quarterly, International Classification, Public National Academic Science - USA, e Organization Science.



Pinto, Adilson Luiz; Matias, Márcio; Moreiro González, José Antonio. Produção brasileira da Ciência da Informação na Web of Science entre 1994 e 2013 e a lista Qualis/Capes da Área. // Ibersid. 10:1 (2016) 51-61. ISSN 1888-0967. 
Figura 3. Centralidade de grau da rede de citação dos periódicos

(Fonte: Dados da pesquisa, 2014. Extraído da Web of Science e Tratado em CiteSpace)

Considerando essa representação visual, os periódicos com círculos maiores são essenciais para a comunidade acadêmica da Ciência da Informação, em especial por serem complementares a tabela II.

Dos oito periódicos mais centrais, três não fazem parte da listagem A1 Qualis/Capes das Ciências Sociais Aplicadas I, no qual a revista Ciência da Informação aparece como B1, por ter suas edições atrasadas devido o fato de a mesma ser gerada em formato impresso, necessitando licitação pública para a impressão de cada número. As outras revistas são DataGramaZero, que é gerenciada desde a sua criação pelo professor Aldo Barreto e; a Journal of Documentation, provavelmente uma das revistas mais representativas da área de Ciência da Informação no mundo, entretanto a mesma não tem publicação de docentes de pós-graduação brasileiros da área, e portanto a mesma não consta na lista Qualis da Área.

\section{Considerações finais}

As bases de dados surgiram para revolucionar o acesso ao conhecimento científico por meio da indexação de periódicos científicos em seus repertórios. Nesse caso, acredita-se que alguns dos títulos internacionais presentes na Web of Science, na qual é possível observar centralidade de grau e frequência, são considerados essenciais para a Ciência da Informação em nível internacional.

Considerando a produtividade de autores, verificou-se que os mais produtivos não estão vinculados aos programas de pós-graduação em Ciência da Informação, como Marcos Andre Gonçalves, Jacqueline Leta e Alberto H. F. Laender. A pós-graduação da área começa a ter destaque a partir da quarta maior produtora, a professora Leilah S. Bufrem.

Da identificação dos periódicos mais citados, pode-se inferir que esses títulos exercem um papel fundamental para a Ciência da Informação brasileira porque figuram como os mais renomados da área, conforme o mapa de centralidade de grau nas quais suas relações são observadas. Segundo a teoria de Bradford, alguns dos jornais que apresentaram maior índice de citação foram: Journal of the American Society Information Science, Information Processing \& Management, College Research Library,
Communication ACM e o Journal of Documentation.

De acordo com a Lei de Lotka, a representação de produtividade dos autores mostra uma produção relevante de autores como Tenopir, Nicholas, Oppenheim, Oder, Rousseau e Egghe, que publicaram em co-autoria com os autores brasileiros. De certa forma, mostra a integração da Ciência da Informação brasileira com referências mundiais na área.

De certa forma, podemos identificar que existem muitas falhas no sistema Qualis/Capes, onde a sua representação se baseia que padrões de publicação e não de uma listagem ideal, onde o que deveria figurar são as revistas indexadas em repositórios internacionais, como as bases multidisciplinares (Web of Science, Scopus e SciELO) e as bases especializadas (LISA, LISTA e ISTA).

Outra coisa que pode ser aplicada, para uma melhoria constante da listagem, seria a análise de citação das publicações, não somente em bases de dados mas em recursos livres, como a gerada pela Publish or Perish (que é derivada do Google Acadêmico); bem como o fator de impacto das revistas, o índice-h das revista e o índice de citação/ano. E não mesmo importante, uma adequação das revistas segundo suas informações no UlrichsWeb, coisa que a área de Ciência da Informação (dentro da listagem das Ciências Sociais Aplicadas I) ainda argumentou e aplicou como padrão inicial de análise das revistas indexadas.

Por fim, acreditamos que a comunidade científica continua buscando construir um modelo de avaliação que seja efetivo, justo e que seja uma representação da qualidade real das publicações científicas para suas respectivas áreas; com isso, pode-se orientar processos de melhoria da qualidade da produção científica e dos seus veículos associados; um modelo que forneça subsídios para orientar o planejamento $e$ as políticas de produção dos programas de pósgraduação e o processo de melhoria da qualidade dos periódicos; e que também estimule continuamente a qualidade das pesquisas, da produção científica dos cursos, dos processos de ensino-aprendizagem e dos profissionais formados pelo sistema brasileiro de pósgraduação. 


\section{Referências}

Andrade, J. B.; Galembeck, F. (2009). Qualis: quo vadis? // Química Nova. 32:1, 5

Capes (2005). Critérios de implantação Qualis: Ciências Sociais Aplicadas. Brasília, setembro.

Capes (2013). Documento de área 2013: Ciências Sociais Aplicadas I. http://qualis.capes.gov.br/webqualis/publico/ documentosDeA-

rea.seam;jessionid=81949B74D43715F79881E62B39B 9C5E8.qualismodcluster-node101? conversationPropagation=begin (2014-04-11).

Capes (2008). Manual WebQualis 3.0. http://www.capes. gov.br/servicos/sala-de-imprensa/36-noticias/2550-cap es-aprova-a-nova-classificacao-do-qualis (2014-04-11).

Capes (2007). Perguntas Mais Freqüentes - Qualis/Capes. http://www.capes.gov.br/servicos/sala-de-imprensa/36noticias/2550-capes-aprova-a-nova-classificacao-do-qua lis (2014-04-11).

Chen, C. (2006). CiteSpace II: detecting and visualizing emerging trends and transiet patterns in scientific literature. // Journal of the American Society for Information Science and Technology. 57:3, 359-377.

Dupont, J.; Dias, L.C. (2008). Renovação do Qualis: exemplo da Química. // Química Nova. 31:6, 1283-1284.

Erdmann, A. L. et al. (2009). A avaliação de periódicos científicos Qualis e a produção brasileira de artigos da área de enfermagem. // Revista Latino-Americana de Enfermagem. 17:3. http://www.scielo.br/pdf/rlae/v17n3/ pt_19.pdf (2014-04-11).

Garvey, W. D. (1979). Communication: the essence of science facilitating information among librarians, scientists, engineers and students. Oxford: Pergamon Press.

Jacon, M. C .M. (2007). Base Qualis e a indução do uso de periódicos da área de Psicologia. // TransInformação. 19:2, 189-197.
Leite, F.; Codato, A. (2013). Autonomização e institucionalização da Ciência Política brasileira: o papel do sistema Qualis-Capes. // Revista de Discentes de Ciência Política da UFSCAR. 1:1, 1-23.

Leydesdorff, L. (2007). Visualization of the Citation Impact Environments of Scientific Journals: An Online Mapping Exercise. // Journal of the American Society for Information Science and Technology. 58:1, 25-38.

Lins, M. P. E.; Pessôa, L. A. M. (2010). Desafios da avaliação de publicações em periódicos: discutindo o novo Qualis da Área Engenharias III. // Revista Brasileira de Pós-Graduação. 7:12, 14-33.

Macias-Chapula, C. A. (1998). O papel da informetria e da cienciometria e sua perspectiva nacional e internacional. // Ciência da informação. 27:2, 134-140.

Población, D. A.; Oliveira, M. (2006). Input e output: insumos para o desenvolvimento da pesquisa. In: población, D. A.; Witter, G. P.; Silva, J. F. M. Comunicação \& produção científica: contexto, indicadores e avaliação. São Paulo: Angellara, 57-80.

Price, J. D. S. (1963). A calculus of science. // International Science and Technology. 15:1, 37-43.

Rocha-E-Silva, M. (2009). O novo Qualis: ou a tragédia anunciada. // Clinics. 64:1, 1-4.

Silva, M. R. (2009). Carta aberta ao Presidente da Capes: o novo Qualis, que não tem nada a ver com a ciência do Brasil. // Pró-Fono Revista de Atualização Científica. 21:4, 3-6.

Spinak, E. (1998). Indicadores cienciométricos. // Ciência da Informação. 27:2, 141-148.

Vlachy, J. (1970). Physics journal in retrospect and comparisons. // Czechoslovak Journal of Physics. 20:1, 501-526.

Enviado: 2015-05-08. Segunda versión: 2016-03-08. Aceptado: 2016-05-19 
